\title{
Nasal trigeminal chemosensitivity across the adult life span
}

\author{
CHARLES J. WYSOCKI and BEVERLY J. COWART \\ Monell Chemical Senses Center, Philadelphia, Pennsylvania \\ and \\ TOMAS RADIL \\ Institute of Physiology, Czech Academy of Sciences, Prague, Czech Republic
}

\begin{abstract}
Individuals can determine the side of the nose that receives an odorant during unilateral presentation (lateralize) if endings of the trigeminal nerve are stimulated. By using psychophysical methods, olfactory detection and trigeminal lateralization thresholds for l-butanol were obtained from 142 individuals ranging in age from 20 to 89 years. Sensitivity in both chemosensory pathways declined with advancing age, especially in people older than 60 years.
\end{abstract}

Although there have been extensive normative studies of human olfaction across the adult life span (e.g., Cowart, 1989; Doty et al., 1984; Wysocki \& Gilbert, 1989), no comparable characterization of nasal irritation is available (Hummel \& Livermore, 2002), although some information on odor/irritant discrimination is available (Laska, 2001). In large part, this reflects the difficulty of assessing trigeminal chemosensitivity in the nose independently of olfaction. Olfactory bipolar neurons serve as distance receptors for ambient chemical stimuli and respond to low concentrations of odorants. Trigeminal free nerve endings, which also are sensitive to mechanical and thermal stimuli, appear to respond primarily to noxious and potentially harmful levels of chemical stimulation (Keverne, Murphy, Silver, Wysocki, \& Meredith, 1986). With few exceptions (carbon dioxide $\left[\mathrm{CO}_{2}\right]$ may be the only one in humans; see the review by Coates, 2001), chemical stimuli that elicit a nasal trigeminal response will also provoke olfactory sensations.

Historically, four approaches to assessing nasal trigeminal function have been utilized in attempts to circumvent the chemosensory confounding between olfactory and trigeminal afferents: testing individuals who lack olfactory function (anosmics), instructing subjects to disregard odor and report only on the irritant components of nasal perception, focusing exclusively on $\mathrm{CO}_{2}$ as a nasal trigeminal stimulus, and a procedure that approximates

Financial support was obtained in part from the National Institute on Deafness and Other Communication Disorders (P50 DC00214 and RO1 DC00298), the Fogarty Center (F05 TW04839) at the National Institutes of Health, and the Eugene Garfield Foundation. Barry Green contributed to the development of the lateralization test protocol. We thank him for suggestions and comments on an earlier version of this paper. Correspondence concerning this report should be addressed to C. J. Wysocki, Monell Chemical Senses Center, 3500 Market Street, Philadelphia, PA 19104 (e-mail: wysocki@monell.org). the one used in this report—namely, direct application of liquid stimuli to the nostril, with subsequent reporting of the location of irritation (Prah \& Benignus, 1984). In 1992, we introduced lateralization as a formal method for assessing intranasal sensitivity to chemical irritants (Wysocki, Green, \& Malia, 1992). This procedure also relies upon localizing (at least to the left or right nostril) the source of irritation in the nose, but stimuli are delivered in vapor, rather than in liquid (Prah \& Benignus, 1984), phase. This procedure appears to be well accepted and provides results that improve upon those obtained from procedures that rely on plastic squeeze bottles (ComettoMuñiz, Cain, Hiraishi, Abraham, \& Gola, 2000).

Sensitivity to suprathreshold concentrations of $\mathrm{CO}_{2}$ was assessed in a few normative studies in which age differences in nasal trigeminal chemosensitivity were examined. A variety of measures were employed, including intensity scaling via magnitude estimation or magnitude matching (Stevens \& Cain, 1986; Stevens, Plantinga, \& Cain, 1982), reflex apnea (Stevens \& Cain, 1986), and measures of chemosensory event-related potential (CSERP) amplitude and latency (Hummel, Barz, Pauli, \& Kobal, 1998). On all of these measures, older subjects were less responsive to nasally presented $\mathrm{CO}_{2}$ than were younger subjects (Hummel et al., 1998; Stevens \& Cain, 1986; Stevens et al., 1982). In the one study that appraised threshold sensitivity to $\mathrm{CO}_{2}$, Stevens and Cain failed to find a significant difference between young and old subjects, even though substantial group differences in these same subjects' responses to suprathreshold $\mathrm{CO}_{2}$ were observed. Because "[t]he failure to obtain a significant young-old difference came contrary to expectations" (p. 325), the authors repeated the evaluations, with the same result; they did, however, note substantial variability in thresholds across subjects.

In short, there is evidence that at suprathreshold levels, nasal trigeminal chemosensitivity declines with age (see, 
also, Laska, 2001), although threshold sensitivity may not. However, there are limits to the interpretation of the results of these studies. Generalizing findings based on responses to a single stimulus is questionable. Although $\mathrm{CO}_{2}$ may not stimulate olfactory receptors (Cain \& Murphy, 1980; but see Kendal-Reed \& Walker, 1997), it may be detectable via weak taste-like sensations in the throat at concentrations below that necessary to elicit nasal trigeminal sensations. Hence, it may be not be appropriate to measure absolute sensitivity of the nasal cavity to this irritant via a traditional detection threshold task (Wysocki et al., 1992; and see Cowart, 1998). The noise added by this factor may have contributed to the variability in nasal thresholds for $\mathrm{CO}_{2}$ reported by Stevens and Cain (1986) and to their failure to observe an age-related decline in this measure. Finally, probably, at least in part, because of practical problems posed by the use of $\mathrm{CO}_{2}$ as a stimulus, virtually all studies of the effects of normal aging on nasal sensitivity to chemosensory irritants have simply compared young with elderly subjects (although Hummel et al., 1998, did include one additional, middleaged group). This type of limited-sample design may be particularly vulnerable to cohort effects and does not allow for estimation of either the onset or the rate of agerelated change.

An interesting distinction between the olfactory and the nasal trigeminal systems appears to lie in their respective abilities to encode spatial information. Skramlik (1925) was the first to provide evidence that subjects cannot identify which side of the nose is receiving a pure olfactory stimulus but can do so readily when the stimulus elicits irritation. His early finding has since been confirmed in several studies (e.g., Kobal, Van Toller, \& Hummel, 1989). Wysocki et al. (1992) introduced an easily administered assessment technique, described in detail by Wysocki, Dalton, Brody, and Lawley (1997), that takes advantage of this distinction to determine not only olfactory detection thresholds, but also nasal lateralization (irritation) thresholds for any volatile compound. The technique makes it feasible to test large numbers of subjects, as well as a wide range of compounds, and perhaps to examine relationships between nasal trigeminal and olfactory sensitivity to specific compounds. It is highly likely that stimulation of free nerve endings or receptors of the trigeminal nerve underlies lateralization thresholds, whereas detection thresholds are most likely based on stimulation of the olfactory epithelium.

In the principal experiment in this report, we used lateralization thresholds to provide what we believe to be the first, decade-by-decade examination of nasal trigeminal chemosensitivity across the adult life span, with 1-butanol (a compound widely used in psychophysical studies of human olfaction; e.g., American Society for Testing and Materials, 1997) serving as the test stimulus. First, however, we will present additional evidence for the failure of the olfactory system to encode spatial information. There has been little disagreement in the literature about the ability of people to localize volatile irritants in the nasal cavity; however, it is not intuitively obvious that olfaction should be incapable of contributing spatial information. Indeed, investigators have argued that pure odorants can be localized (Békésy, 1964; Bellas, Novelly, \& Eskenazi, 1989; Jiang \& Rouby, 1992).

\section{PRELIMINARY EXPERIMENTS}

The goal of two of these preliminary experiments was to provide further tests of the hypothesis that a nonirritating odorant cannot be lateralized and, thus, that the lateralization threshold is a measure of nasal trigeminal responsiveness to volatile compounds that is independent of the odorant's effect on the olfactory system. Since the methodology for determining detection thresholds differed slightly from that used to determine lateralization thresholds, a third experiment was performed to compare and contrast these methods.

\section{Method, Results, and Discussion}

Six women (average age, $35 \pm 15.2[S D]$ years) and 4 men (average age, $41 \pm 15.5[S D]$ years) were given two polypropylene squeeze bottles (glass is now preferred and was used in Experiment 2) with Teflon nosepieces that fit into their nostrils. The nosepieces were custom-built and were washed and sterilized after each use. Odorized bottles contained phenylethyl alcohol (PEA, 3.2\%; $10 \mathrm{ml}$ ), a stimulus that, when delivered in vapor phase, does not appear to elicit nasal trigeminal responses in humans (Cui \& Evans, 1997; Doty et al., 1978; Kobal \& Hummel, 1991; cf. Prah \& Benignus, 1984, who do report trigeminal-like perception when liquid PEA is applied directly to the nasal epithelium). The comparison bottle contained only $10 \mathrm{ml}$ of glycerol, the solvent. The headspace in these bottles $(270 \mathrm{ml})$ was used to stimulate both sides of the nose-independently and simultaneously during the same inspiration. The side of stimulation and the order of administration of stimuli were randomized throughout. The task of the subject was to decide which nostril was stimulated with PEA.

The subjects received 40 lateralization trials (20 per nostril) with PEA versus the blank to establish a baseline for percentage correct (mean $\pm S E M=51.5 \% \pm 3.2 \%$ ). Subsequently, the individuals received 50 trials, were informed about the side of stimulation prior to the trial, and were instructed to turn their head in the direction of the stimulus as an added motor component of the learning task. After this training, the subjects were again tested in 40 trials, without foreknowledge of the side to be stimulated by PEA. The results indicated no significant improvement; individuals again failed to lateralize the odorant (mean $\pm S E M=56.5 \% \pm 5.0 \%$ correct) and showed no improvement over baseline $[t(9)=1.2, p>.25]$.

In a second experiment, 4 women (average age, $34 \pm$ $10.0[S D]$ years) and 1 man (62 years) were each given 100 lateralization trials with PEA $(3.2 \% \mathrm{v} / \mathrm{v})$ versus the diluent in the same delivery apparatus. After each trial, the subjects were informed whether they were correct and were presented with the two bottles in the same spatial arrangement to confirm the condition. If lateraliza- 


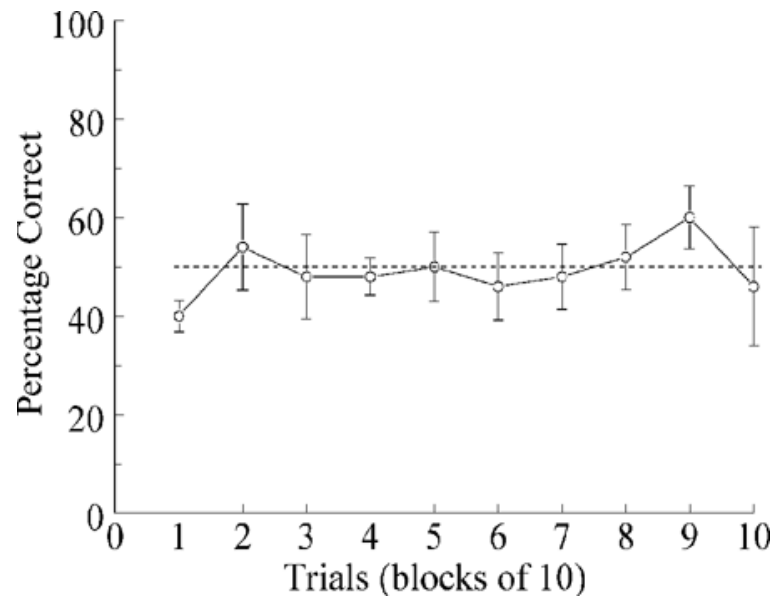

Figure 1. Results of 100 lateralization trials from 5 subjects, using phenylethyl alcohol as a stimulus. The horizontal dotted line represents chance performance.

tion of PEA was possible, albeit difficult, some learning should be evident over the course of the 100 trials. The results (Figure 1) indicate that the individuals did not learn to lateralize the odor over the training session.

We have performed analogous experiments with vanillin and lyral, two additional putative "pure" odorants, with similar results—namely, the subjects failed to lateralize the stimulus even when the vapor phase of neat materials was used. These results and data from other laboratories (e.g., Roscher, Glaser, Hummel, \& Kobal, 1996) confirm that apparently pure olfactory stimuli do not provide sufficient spatial information to be lateralized, even when subjects are given extensive practice in the task. The data also support the interpretation that the ability to lateralize volatile compounds provides an objective measure of nasal trigeminal chemosensitivity that can be used to assess the sensitivity of the system irrespective of the olfactory attributes of the stimulus.

The method employed for determining detection thresholds in the primary experiment (below) relies upon comparisons of sequential pairs of stimulus bottles. In a forced-choice trial, the first of a pair of bottles is sniffed (one in each nostril), and then a second pair is presented (again, one in each nostril). One of the pairs of bottles contains an odorant, whereas the other pair contains blanks. The task of the subject is to choose the pair that contains the odorant. For determining lateralization thresholds, a single pair of bottles is presented (one per nostril), and the task of the subject is to determine which nostril is stimulated by the odorant/irritant. It is possible that this slight difference in methodology could impact on direct comparisons between detection and lateralization thresholds. For this reason, a third experiment was performed, in which lateralization thresholds were determined in two ways: For one method, two pairs of bottles were used in each trial (one pair contained two blanks and the other pair had l-butanol vs. a blank), and for the other, a single pair (butanol vs. blank) was relied upon. Results from tests with 10 volunteers ( 8 males, average age of $34 \pm 5.4$ years; 2 females, 33 and 51 years) revealed that lateralization thresholds determined in the manner used in the primary experiment (below), in which two bottles were used (one blank vs. odorant) did not differ from those obtained when two pairs of bottles (two blanks vs. one blank plus butanol) were used (Radil $\&$ Wysocki, 2000, and unpublished data).

\section{PRIMARY EXPERIMENT}

In this experiment, we measured lateralization thresholds to assess nasal trigeminal chemosensitivity over the adult life span. Butanol was selected as the stimulus because, within the chemosensory research community, it has a long history of use as a routine test stimulus, both as an olfactory stimulus (e.g., Stevens \& Cain, 1986) and, at concentrations comparable to those used by us, as a nasal irritant (Cain, 1976). In addition to lateralization thresholds, traditional olfactory detection thresholds were assessed so that the relationship between the two thresholds could be examined across the human age span.

\section{Method}

Subjects. Healthy individuals $(N=142)$, ranging from 20 to 89 years of age, were recruited from the Monell Center, University of Pennsylvania, and Drexel University communities, from local group residences, and by advertisements. There were 10 males and 10 females within each decade of age, with 1 additional male in his $30 \mathrm{~s}$ and 1 in his 40s. Three racial groups were representednamely, African Americans $(n=23)$, Asians $(n=5)$, and Caucasians $(n=114)$. Among the subjects were 31 cigarette smokers (32\% African American, 20\% Asian, 18\% Caucasian). Individuals were paid \$20 plus travel and other expenses for their participation.

Procedure. All the volunteers were tested at the Monell Center. After demographic information (age, sex, race, smoking history, etc.) was obtained, a self-report on olfactory acuity on a 5-point scale, ranging from 1 (excellent) to 5 (poor), and informed consent, the subjects underwent sensitivity testing (detection and lateralization thresholds) for 1-butanol (Fisher).

Tertiary dilutions of butanol from neat were prepared in nominally "odorless," light, white mineral oil, which also served as the blank. Previous use of this diluent revealed that slight odorous contaminants could be detected in some batches. Hence, routinely for all batches of mineral oil, it was filtered through a column of silica gel to eliminate these potential contaminants prior to use.

Each dilution series contained 26 steps, covered a range from $100 \%$ (Dilution Step 0) to $1.18 \times 10^{-10 \%}$ (Step 25) and was used within 3 weeks of preparation. Stimuli, $10 \mathrm{ml}$ each, were contained in clean $280-\mathrm{ml}$ glass bottles, which were capped with plastic screw-tops. Each top had two holes, through which lengths of Teflon tubing were inserted. One tube, which provided access of room air to the bottle, penetrated the cap and terminated just above the stimulus. The other tube extended from the cap and was topped with a Teflon nosepiece, which, during testing, was inserted into a nostril. It was through this nosepiece and another positioned simultaneously in the other nostril that the subject sniffed the headspace above the odorant or the blank (nosepieces were of the same type as those used in the preliminary experiments, which were washed and sterilized between use).

The subjects were evaluated in a single 45-min session, during which two detection and two lateralization thresholds were obtained. Detection thresholds were obtained by using a two-alternative forced-choice modified staircase method (Wysocki, Cowart, \& Varga, 


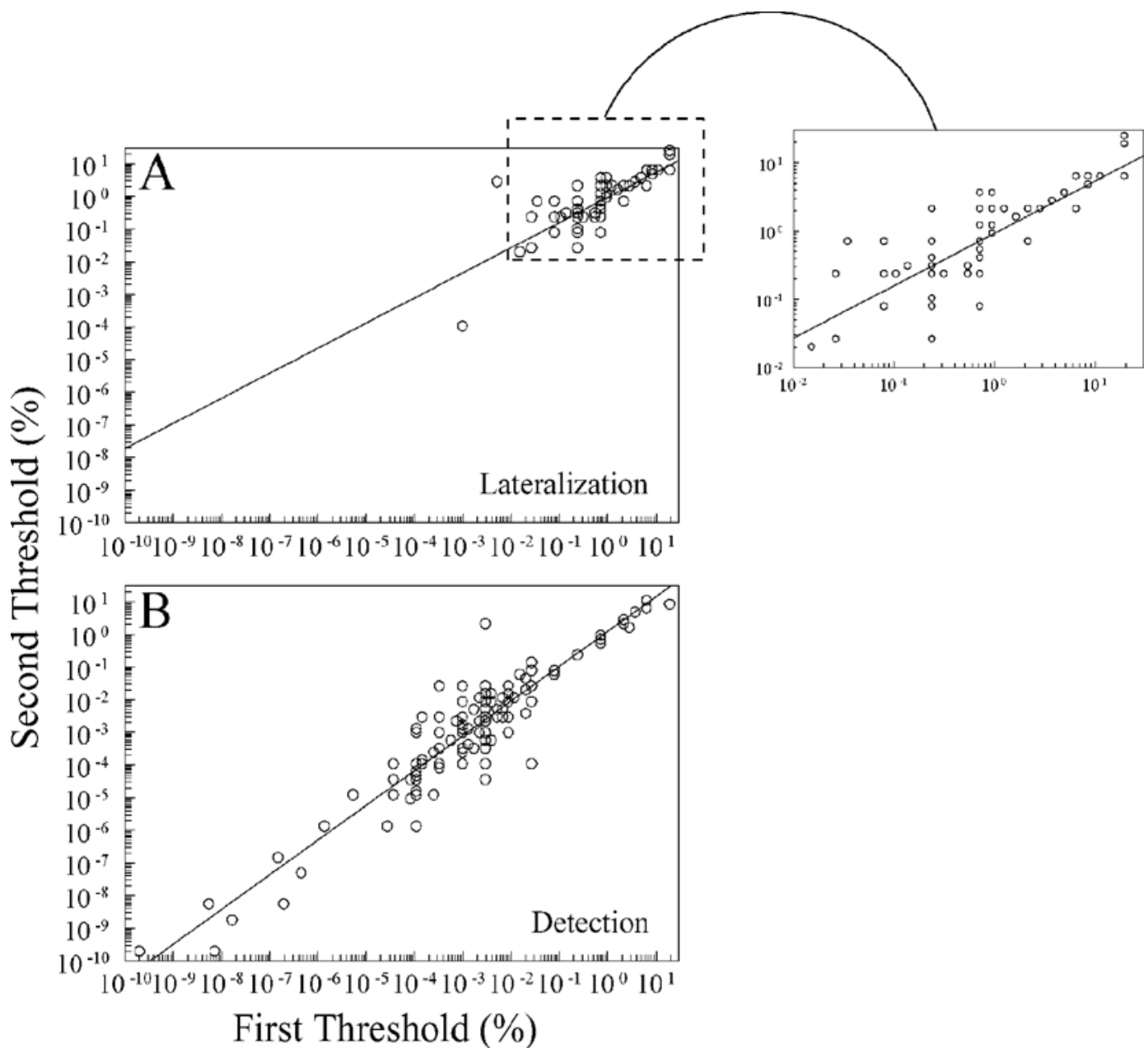

Figure 2. Results of two threshold measures for lateralization (A) and detection (B) of butanol performed in the same test session. For direct comparisons of thresholds, the axes $\left(\log _{10}\right.$ of the percent of butanol in solution) are the same in $A$ and $B$ (note the greater variation in detection thresholds than in lateralization thresholds). The blowup of A captures the 1,000-fold range in which most lateralization thresholds occurred.

1997). On the first trial, the subjects were given two sets of bottles, one consisting of two blanks, the other of one blank and $1.69 \times$ $10^{-3} \%$ butanol (Dilution Step 10). The set of bottles that was presented first on each trial was determined randomly; however, all the individuals received the same sequence. Upon receiving the pair of bottles, the subjects inserted the Teflon nosepieces into each nostril. After both sets of bottles had been sampled, the subjects indicated which set had contained the odorant. If the odorant pair was correctly identified in three consecutive trials, the concentration was decreased by two dilution steps. If an incorrect answer was given, the concentration of butanol was increased by one dilution step (there was a 30 -sec delay between trials). Subsequent changes in dilution steps by single steps required only two consecutive correct choices and continued until five reversals (an increase in concentration followed by a decrease in concentration, or vice versa) had occurred. One additional contingency also had to be met: The distance between any two reversals could not exceed three dilution steps. If this contingency was violated, testing continued until four additional valid reversals were obtained. The mean of the dilution steps of the final four reversals, converted to percent in solution, was defined as the detection threshold.
After a 5-min rest, a lateralization threshold was obtained using a similar two-alternative forced-choice modified, staircase method (Wysocki et al., 1997). During each trial, the subjects were handed two bottles. One bottle was a blank, whereas the other contained butanol. Trials began with $0.41 \%$ butanol (Dilution Step 5). The subjects simultaneously placed the Teflon nosepieces into their nostrils and inhaled. After removing the nosepieces, the subjects indicated whether they thought the stimulus had been presented to the left or to the right nostril. If a subject correctly identified the nostril receiving butanol in two consecutive trials, its concentration was decreased by one dilution step. If the subject was incorrect on any trial, the concentration was increased by a dilution step. The sequence ended after the subject had made at least five valid reversals in concentration level. One additional contingency also had to be met: The distance between any two reversals could not exceed three dilution steps. If this contingency was violated, testing continued until four additional valid reversals were obtained. The mean of the dilution steps of the final four reversals, converted to percent in solution, was defined as the detection threshold.

After another 5-min rest, another detection threshold was obtained using procedures identical to those used to obtain the first detection 


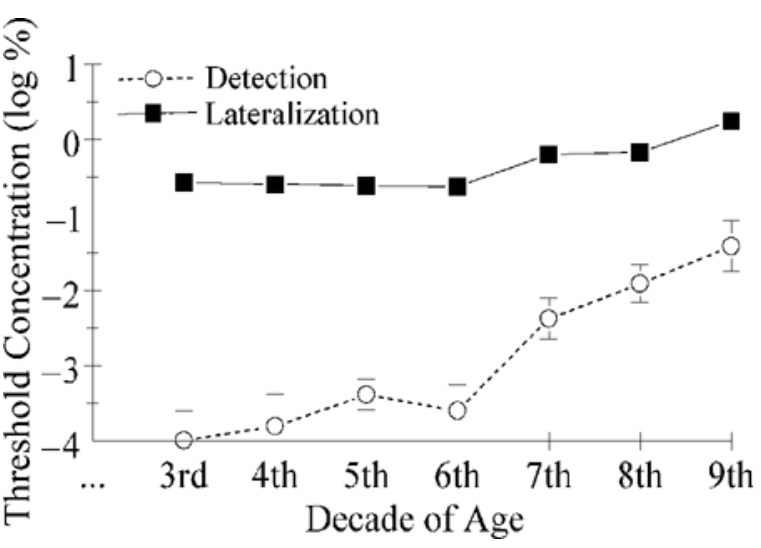

Figure 3. Average detection and lateralization thresholds, expressed as $\log _{10}$ of the percent in solution, $\pm S E M$ (those for lateralization are buried within the marker and cannot be seen), across the age range of subjects, grouped by decade.

threshold, with the exception that the starting step was the concentration just below the calculated detection threshold determined in the first procedure. After yet another 5-min rest, another lateralization threshold was obtained using procedures identical to those used to obtain the first lateralization threshold, again with the exception that the starting step was the concentration just below the calculated lateralization threshold determined in the first procedure.

Data analysis. Thresholds, expressed as percent in solution, were not normally distributed; hence, data were $\log _{10}$ normalized prior to analyses. Where appropriate, analyses of variance (ANOVAs) or covariance (ANCOVAs) using a repeated measures design, regression analyses, or bivariate or partial correlations were applied to the data (SPSS for Windows, Rel 6.1.3). Because multiple variables were evaluated in some analyses and statistical tests were applied subsequent to an overall analysis, the level of significance was typically set at $p<.01$.

\section{Results}

In an overall repeated measures ANCOVA (threshold type [2] $\times$ trials [2] $\times$ gender [2] $\times$ race [3] $\times$ smoking status [2]), with age entered as a covariate, gender, race, and smoking status were not significant factors $[F(1,130)=2.74, p=.10 ; F(1,130)=1.68, p=.19$, and $F(1,130)=0.39, p=.53$, respectively $]$ and did not enter into any significant interactions. The regression on age was significant $[F(1,130)=53.35, p<.001]$, and there was a significant difference between detection and lateralization thresholds $\left[1.21 \times 10^{-3 \%}\right.$ vs. $4.12 \times 10^{-1 \%}$, respectively; $F(1,131)=56.89, p<.001]$. Repeated testing of each threshold did not make a significant contribution to overall variance $[F(1,131)=0.02, p=.88$; the two repeated measures factors-namely, the type of threshold and the test-retest of the threshold-did not significantly interact with each other: $F(1,131)=0.16$, $p=.69]$.

Repeated tests of each threshold demonstrated significant reliability $(p<.001)$ of the procedure for both thresholds, both before controlling for the effects of age (detection, $r=.93$; lateralization, $r=.76$; see Figure 2, bottom and top, respectively) and after (detection, partial $r=.90 ;$ lateralization, partial $r=.71$ ). Because repeated testing did not make a significant contribution to overall variance but did have significant test-retest reliability, the two analogous thresholds $\left(\log _{10}\right.$ of percentage of dilution) were averaged.

The averages were then reanalyzed in a repeated measures ANOVA after collapsing the age of the subjects into decades. The analysis again revealed a significant effect of age (decade [7 levels]) on the thresholds; with advancing age, both detection and lateralization thresholds increased - that is, the sensory systems became less sensitive $[F(6,135)=12.31, p<.001]$. Although detection thresholds were lower than lateralization thresholds across the life span $[F(6,135)=465.31, p<.001]$, there was a significant interaction between the two thresholds with advancing age [see Figure $3 ; F(6,135)=5.35, p<$ $.001]$. Inasmuch as the direct comparison between the two thresholds revealed a striking difference between them, in some of the subsequent analyses, each was treated individually.

In separate regression analyses of the thresholds (averages of the $\log _{10}$ test and retest values), age was a significant contributor to variation for both detection $[F(1,140)=$ $50.59, p<.001 ; r=.51$; see Figure 4 , bottom] and lateralization $[F(1,140)=35.67, p<.001 ; r=.45$; see Figure 4, top]. Individual ANOVAs revealed that, with advancing age, thresholds from individuals increased across decades (see Figure 3). The average detection threshold for individuals in their 20s was Dilution Step $12.5( \pm 0.8)$, which increased across decades to reach $7.2( \pm 0.7)$ for octogenarians $[F(6,135)=9.69, p<.001]$. The average lateralization threshold for individuals in their 20 s was Dilution Step $5.4( \pm 0.4)$, which increased to $3.7( \pm 0.3)$ for octogenarians $[F(6,135)=8.67, p<.001]$. Post hoc difference contrasts, which assessed the significance of any difference between the mean value for a decade and the average threshold of all those who were younger-for example, threshold for the 7 th decade of age versus the average value calculated from those whose age was $20-59$ yearsrevealed that both detection and lateralization thresholds were significantly elevated $(p<.002)$ in each of the three decades for individuals $\geq 60$ years old.

The correlation between the average log-detection and average log-lateralization thresholds was significant $(r=$ $.52, p<.001)$ and remained so after controlling for the effects of age (partial $r=.38, p<.001$ ). Data from individuals $\geq 60$ years old account for this correlation; controlling for the effects of age, the partial correlation $(r=$ .69) for people $\geq 60$ years was significant $(p<.001)$, whereas for individuals $<60$ years old, the partial correlation was not significant $(r=.09, p>.40)$. In even more focused analyses, bivariate correlations between the two thresholds for people in each of the decades for individuals $<60$ years of age were not significant, but within each of the three remaining decades they were $(p<.01)$.

In the overall analysis, neither race nor smoking status made a significant contribution to overall variation; however, unbalanced sample sizes may have affected the 


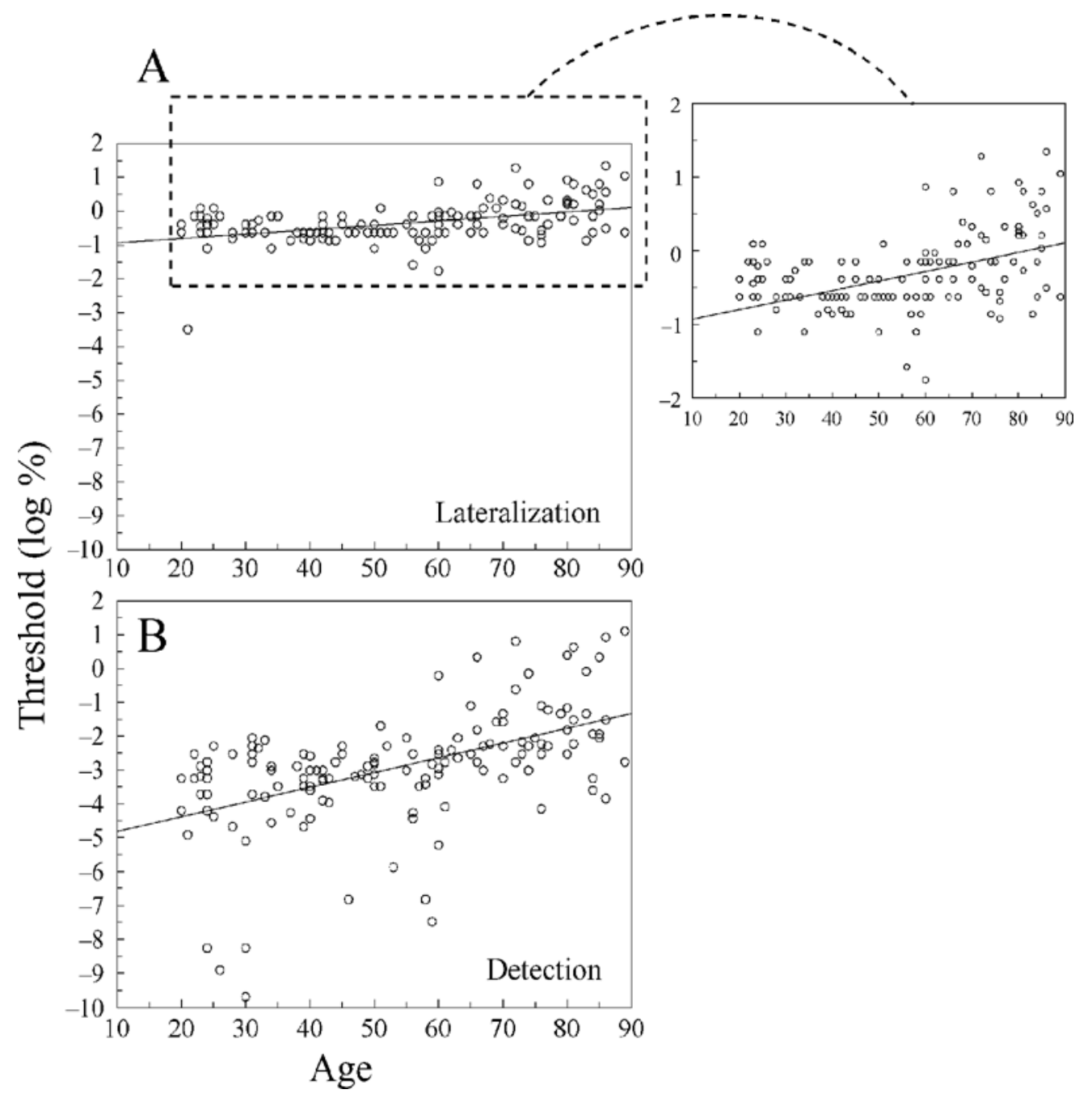

Figure 4. Scatterplot of the average individual lateralization (A) and detection (B) thresholds, $\operatorname{expressed}$ as $\log _{10}$ of the percent in solution, across the age range of subjects. The calculated regression line is indicated within the points. For direct comparisons of thresholds, the vertical axes are the same in panels $A$ and $B$. The blowup of $A$ captures the 1,000 -fold range in which most lateralization thresholds occurred.

outcome. To adjust for this, the 23 African Americans were matched by gender and age to Caucasians. Reanalyses, with and without gender as a factor, again showed no significant contribution of race to either detection or lateralization threshold ( $p>.60$ in each case). In the reanalyses of the potential effects of cigarette smoking on sensitivity to butanol, ex-smokers were eliminated from consideration, and the 31 smokers were matched by age, sex, and race (where possible) to nonsmokers. The data were then analyzed with and without gender as a factor. Smoking affected neither detection nor lateralization threshold ( $p>.05$ in each case).

After eliminating the only subject whose self-rating of olfaction was poor, two-way ANCOVAs, with olfactory self-rating (four levels) and gender (two levels) as grouping factors and age as a covariate, revealed that selfperception of olfactory acuity was not a significant con- tributor $(p>.05)$ to overall variation in either the detection $[F(3,132)=2.36]$ or the lateralization $[F(3,136)=2.28]$ threshold.

\section{GENERAL DISCUSSION}

Both olfactory and trigeminal afferents are stimulated by most volatile compounds. The initial processing of odor information originates from the former, whereas the perception of irritation from within the nose is conveyed via the latter. On the basis of the premise that irritation, but not odor, produced by volatile compounds can be localized (Skramlik, 1925), we and others (Roscher et al., 1996; Wysocki, Dalton, et al., 1997; Wysocki et al., 1992) devised methods that rely upon an ability to lateralize stimuli to assess sensitivity of the nasal trigeminal system to volatile compounds. These methods now 
allow us to determine the concentrations at which trigeminal stimulation becomes functionally relevant for lateralization when an odorant is sniffed.

Although a mean difference between olfactory and trigeminal thresholds for butanol is evident from the data (Figure 4), so too is the striking contrast in their respective variations. The range in detection thresholds exceeded 10 orders of magnitude, suggesting profound individual differences in olfactory sensitivity; lateralization thresholds were restricted to roughly 4 orders of magnitude. In part, this may have been due to the ranges of concentrations per se. At the concentration levels necessary to obtain a lateralization threshold, the change in concentration across a single step is much more (in terms of molecular mass) than that noted at concentrations needed for detection. In addition, age per se does not account for all of this variation. Indeed, a comparison of averages within decades reveals roughly a $2.5 \log _{10}$ difference in olfactory detection thresholds across the life span, which approximates the degree of shift noted in Cain and Stevens (1989), and less than an order-of-magnitude change in lateralization thresholds. Without actually measuring thresholds, Kendal-Reed, Walker, Morgan, LaMacchio, and Lutz (1998), who used a statistical approach that relied upon sampling of data, argued that "within- and between-participant variation in (olfactory) sensitivity is far less than is commonly reported" (p. 71) and that differences of the magnitude noted by us and by others result from methodological issues, which could be overcome by using precision olfactometry. Undoubtedly, methodology makes some contribution to observed variation; however, given the test-retest reliability noted above, we suggest that olfactometry per se will not eliminate the variation reported by us and others to the extent suggested by Kendal-Reed et al. (1998).

Although inconsistent, reported gender differences suggest that women are more sensitive to nasal trigeminal stimuli than are men. Cometto-Muñiz and Noriega (1985) noted that women rated $\mathrm{CO}_{2}$ in the nose, but not in the mouth, as significantly more intense than did men. Medina and Cain (1982) found the apnea reflex to be elicited at significantly lower concentrations for women than for men, and Shusterman and Balmes (1997) reported a lower $\mathrm{CO}_{2}$ threshold for women than for men. On the other hand, neither Medina and Cain nor Stevens et al. (1982) observed gender differences in ratings of nasal irritation elicited by $\mathrm{CO}_{2}$, and although Hummel et al. (1998) reported a tendency for women to exhibit higher amplitude CSERP in response to $\mathrm{CO}_{2}$ in the nose, this was not statistically significant. We also observed no gender differences in lateralization thresholds for butanol.

As is indicated by the correlations, nasal sensitivity to the irritation of butanol appears to be linked to olfactory sensitivity to this odorant, at least in individuals $\geq 60$ years of age. This linkage is reflected in an age-related diminution in sensitivity in both systems - changes that were not accurately reflected in individual self-reports of olfactory acuity. Why these thresholds would be cor- related in older, but not younger, subjects remains to be determined. The observation that a decline in olfactory sensitivity is accompanied by a decrease in sensitivity to intranasal irritants is, however, consistent with other clinical findings - namely, that hyposmia and anosmia apparently are associated with a decrease in nasal sensitivity to the irritation of butanol (Wysocki, Cowart, \& Varga, 1997) and other stimuli (Hummel et al., 1996). Perhaps, even though olfaction per se does not convey spatial information, its activation facilitates responses to irritants.

Although there now are numerous publications attesting to a decline in olfactory capabilities with advancing age (e.g., Cain \& Stevens, 1989; Cowart, 1989; Doty et al., 1984; Wysocki \& Gilbert, 1989), the work presented here is the first systematic exploration of age-related influences on sensitivity to an intranasal irritant across the life span. Whether the shifts in sensitivity noted for butanol extend to other nasal irritants (which seems likely; see Hummel et al., 1998) and whether the rate of decline in sensitivity parallels that for butanol (Cain \& Stevens, 1989; cf. Wysocki \& Gilbert, 1989) remain to be determined. What does appear to occur is a loss with age of discrimination of irritant qualities across different types of odorants/irritants (Laska, 2001).

\section{REFERENCES}

American Society for Testing and Materials (1997). Standard Practices for Referencing Suprathreshold Odor Intensity [Standard E544-75 (1988) e1]. West Conshohocken, PA: Author.

BÉKÉSY, G. voN (1964). Olfactory analogue to directional hearing. Journal of Applied Physiology, 19, 369-373.

Bellas, D., Novelly, R. A., \& Eskenazi, B. (1989). Olfactory lateralization and identification in right hemisphere lesion and control patients. Neuropsychologia, 27, 1187-1191.

CAIN, W. S. (1976). Olfaction and the common chemical sense: Some psychophysical contrasts. Sensory Processes, 1, 57-67.

CAIN, W. S., \& MurPhy, C. L. (1980). Interactions between chemoreceptive modalities of odour and irritation. Nature, 284, 255-257.

Cain, W. S., \& Stevens, J. C. (1989). Uniformity of olfactory loss in aging. In C. L. Murphy, W. S. Cain, \& D. M. Hegsted (Eds.), Chemical senses and nutrition in aging (Annals of the New York Academy of Sciences, Vol. 561,pp. 29-38). New York: New York Academy of Sciences.

CoAtes, E. L. (2001). Olfactory $\mathrm{CO}_{2}$ chemoreceptors. Respiration Physiology, 129, 219-229.

Cometto-Muñz, J. E., Cain, W. S., Hiraishi, T., Abraham, M. H., \& GoLA, J. M. R. (2000). Comparison of two stimulus-delivery systems for measurement of nasal pungency thresholds. Chemical Senses, 25, 285-291.

Cometto-MuñIz, J. E., \& Noriega, G. (1985). Gender differences in the perception of pungency. Physiology \& Behavior, 34, 385-389.

CowART, B. J. (1989). Relationships between taste and smell across the adult life span. In C. L. Murphy, W. S. Cain, \& D. M. Hegsted (Eds.), Chemical senses and nutrition in aging (Annals of the New York Academy of Sciences, Vol. 561, pp. 39-55). New York: New York Academy of Sciences.

CowArT, B. J. (1998). The addition of $\mathrm{CO}_{2}$ to traditional taste solutions alters taste quality. Chemical Senses, 23, 397-402.

CuI, L., \& Evans, W. J. (1997). Olfactory event-related potentials to amyl acetate in congenital anosmia. Electroencephalography \& Clinical Neurophysiology, 102, 303-306.

Doty, R. L., Brugger, W. E., Jurs, P. C., Orndorff, M. A., Snyder, P. J., \& Lowry, L. D. (1978). Intranasal trigeminal stimulation from odorous volatiles: Psychometric responses from anosmic and normal humans. Physiology \& Behavior, 20, 175-185. 
Doty, R L., Shaman, P., Applebaum, S. L., Giberson, R., Siksorski, L., \& Rosenberg, L. (1984). Smell identification ability: Changes with age. Science, 22, 1441-1443.

Hummel, T., Barz, S., Lötsch, J., Roscher, S., Kettenmann, B., \& KoвAL, G. (1996). Loss of olfactory function leads to a decrease of trigeminal sensitivity. Chemical Senses, 21, 75-79.

Hummel, T., Barz, S., Pauli, E., \& Kobal, G. (1998). Chemosensory event-related potentials change with age. Electroencephalography \& Clinical Neurophysiology, 108, 208-217.

Hummel, T., \& Livermore, A. (2002). Intranasal chemosensory function of the trigeminal nerve and aspects of its relation to olfaction. International Archives of Occupational \& Environmental Health, 75, 305-313.

JiAnG, T., \& Rouby, C. (1992). Human capacity to localize mononarinal odor stimuli. Chemical Senses, 17, 845-846.

Kendal-Reed, M., \& Walker, J. C. (1997). Sensory and respiratory responses of normals and anosmics to $\mathrm{CO}_{2}$ presented by precision olfactometer. Chemical Senses, 22, 717.

Kendal-Reed, M., Walker, J. C., Morgan, W. T., LaMacchio, M., \& LUTz, R. W. (1998). Human responses to propionic acid: I. Quantification of within- and between-participant variation in perception by normosmics and anosmics. Chemical Senses, 23, 71-82.

Keverne, E. B., Murphy, C. L., Silver, W. L., Wysocki, C. J., \& Meredith, M. (1986). Non-olfactory chemoreceptors of the nose: Recent advances in understanding the vomeronasal and trigeminal systems. Chemical Senses, 11, 119-133.

Kobal, G., \& Hummel, T. (1991). Olfactory evoked potentials in humans. In T. V. Getchell, R. L. Doty, L. M. Bartoshuk, \& J. B. Snow, Jr. (Eds.), Smell and taste in health and disease (pp. 255-275). New York: Raven.

Kobal, G., Van Toller, S., \& Hummel, T. (1989). Is there directional smelling? Experientia, 45, 130-132.

LASKA, M. (2001). Perception of trigeminal chemosensory qualities in the elderly. Chemical Senses, 26, 681-689.

Medina, M. R. G., \& CAIN, W. S. (1982). Bilateral integration in the common chemical sense. Physiology \& Behavior, 29, 349-353.

Prah, J. D., \& Benignus, V. A. (1984). Trigeminal sensitivity to con- tact chemical stimulation: A new method and some results. Perception \& Psychophysics, 35, 65-68.

RADIL, T., \& WYSOCKI, C. J. (2000). Butanol detection and lateralization: Conscious and unconscious mechanisms [Abstract]. Chemical Senses, 25, 624.

Roscher, S., Glaser, C., Hummel, T., \& Kobal, G. (1996). An easy method for separating olfactory from trigeminal stimulation. Chemical Senses, 21, 492-493.

Shusterman, D., \& Balmes, J. (1997). Measurement of nasal irritant sensitivity to pulsed carbon dioxide: A pilot study. Archives of Environmental Health, 52, 334-340.

SKRAMLIK, E voN (1925). Über die Lokalisation der Empfindungen bei den niederen Sinnen [On the localization of sensation in the lower senses]. Zeitschrift für Sinnesphysiologie, 56, 69-140.

Stevens, J. C., \& CAIN, W. S. (1986). Aging and the perception of nasal irritation. Physiology \& Behavior, 37, 323-328.

Stevens, J. C., Plantinga, A., \& Cain, W. S. (1982). Reduction of odor and nasal pungency associated with aging. Neurobiology of Aging, 3, 125-132.

Wysocki, C. J., Cowart, B. J., \& VARGA, E. (1997). Nasal-trigeminal sensitivity in normal aging and clinical populations [Abstract]. Chemical Senses, 22, 826.

Wysocki, C. J., Dalton, P., Brody, M. J., \& LaWley, H. J. (1997). Acetone odor and irritation thresholds obtained from acetone-exposed factory workers and from control (occupationally unexposed) subjects. American Industrial Hygiene Association Journal, 58, 704-712.

Wysocki, C. J., \& GilberT, A. N. (1989). National Geographic smell survey: Effects of age are heterogenous. In C. L. Murphy, W. S. Cain, $\&$ D. M. Hegsted (Eds.), Chemical senses and nutrition in aging (Annals of the New York Academy of Sciences, Vol. 561, pp. 12-28). New York: New York Academy of Sciences.

Wy socki, C. J., Green, B. G., \& Malia, T. P. (1992). Monorhinal stimulation as a method for differentiating between thresholds for irritation and odor. Chemical Senses, 17, 722-723.

(Manuscript received September 2, 1998; revision accepted for publication May 9, 2002.) 